\title{
The endoplasmic reticulum membrane protein complex localizes to the mitochondrial - endoplasmic reticulum interface and its subunits modulate phospholipid biosynthesis in Trypanosoma brucei
}

Advaitha Iyer ${ }^{1,2}$, Moritz Niemann ${ }^{3,4}$, Luce Farine ${ }^{1}$, Mauro Serricchio ${ }^{1}$, André Schneider ${ }^{3}$, Peter Bütikofer ${ }^{1 \#}$

${ }^{1}$ Institute of Biochemistry and Molecular Medicine, University of Bern, Bern, Switzerland

${ }^{2}$ Graduate School for Cellular and Biomedical Sciences, University of Bern, Bern, Switzerland

${ }^{3}$ Department of Chemistry, Biochemistry and Pharmaceutical Sciences, University of Bern, Bern, Switzerland

${ }^{4}$ present address: EMBL Imaging Centre, Meyerhofstrasse 1, 69117 Heidelberg, Germany

${ }^{\#}$ Correspondence to:

Peter Bütikofer, PhD, Institute of Biochemistry and Molecular Medicine, University of Bern, Bühlstrasse 28, 3012 Bern, Switzerland / E-mail: peter.buetikofer@ibmm.unibe.ch

Running title: The trypanosomal EMC modulates phospholipid biosynthesis

Keywords: EMC, phospholipid biosynthesis, T. brucei, mitochondrial ER-interface 


\begin{abstract}
The endoplasmic reticulum membrane complex (EMC) is a versatile complex that plays a key role in membrane protein biogenesis in the ER. Deletion of the complex has wide-ranging consequences including ER stress, disturbance in lipid transport and organelle tethering, among others. Here we report the function and organization of the evolutionarily conserved EMC (TbEMC) in the highly diverged eukaryote, Trypanosoma brucei. We show that the TbEMC is composed of 9 subunits that are present in a high molecular mass complex localizing to the mitochondrial-endoplasmic reticulum interface. Knocking out or knocking down of single TbEMC subunits led to growth defects of T. brucei procyclic forms in culture. Interestingly, we found that depletion of individual TbEMC subunits lead to disruption of de novo synthesis of phosphatidylcholine (PC) or phosphatidylethanolamine (PE), the two most abundant phospholipid classes in T. brucei. Downregulation of TbEMC1 or TbEMC3 inhibited formation of PC while depletion of TbEMC8 inhibited PE synthesis, re-emphasizing the role of the EMC in phospholipid synthesis. In addition, we found that in TbEMC7 knock-out parasites, TbEMC3 is released from the complex, implying that TbEMC7 is essential for the formation or the maintenance of the TbEMC. The organization of the TbEMC and its role in phospholipid metabolism in T. brucei may represent ancestral functions.
\end{abstract}




\section{Introduction}

The endoplasmic reticulum (ER) is a vast multifarious membrane system that plays a key role in lipid and protein synthesis in a cell. It is known to house many protein complexes that aid its function, including the conserved ER membrane protein complex (EMC) ${ }^{1}$. The EMC was first described more than a decade ago in Saccharomyces cerevisiae as a complex composed of 6 proteins $^{2}$ and is now believed to contain 8 subunits localizing exclusively to the $\mathrm{ER}^{3}$. Since then, EMCs have been identified in every major eukaryotic lineage ${ }^{1}$. The wide distribution of the EMCs and the high degree of sequence conservation between the corresponding subunits of the complex underline their functional importance. The EMC appears to be functionally pleotropic, with roles in cellular processes ranging from membrane protein insertion, viral protein stabilization and ER stress to lipid transport ${ }^{2,4-8}$.

Work in $S$. cerevisiae implied that the EMC tethers the ER to the mitochondria ${ }^{7}$. Simultaneous deletion of multiple subunits of the EMC lead to defects in phospholipid trafficking from the ER to the mitochondria, resulting in impaired mitochondrial function ${ }^{7}$. Subsequently, the role of the EMC in phospholipid metabolism has received little additional attention. Instead, more recent studies have focused on the role of the EMC in the biogenesis of multipass membrane proteins ${ }^{9}$ and the association of its subunits with cell surface membrane proteins $^{10-12}$, indicating that it may act as transmembrane domain (TMD) chaperone ${ }^{4}$ or insertase $^{13}$. In mammalian cells, insertion of the ER resident enzyme, squalene synthase, was found to depend on a functional $\mathrm{EMC}^{13}$ and in reconstitution experiments, a purified 10 component EMC facilitated membrane insertion of squalene synthase and other reporter proteins, indicating that the EMC mediates insertion of proteins with low hydrophobicity ${ }^{13}$. Furthermore, a mass spectrometry-based proteomic analysis identified a list of EMC subunitdependent proteins containing TMDs with polar and/or charged residues ${ }^{14}$. In structural studies, the human EMC has been suggested to mediate membrane insertion of proteins 
containing positively charged amino acids within their $\mathrm{TMD}^{15}$. The model implicates EMC3 in the capture and targeting of the substrate to the membrane. Subsequently, substrate insertion may be achieved via a hydrophilic vestibule composed of EMC3 and EMC6, whereby a local thinning of the membrane and a positively charged TMD patch in the bilayer further assist substrate insertion. The unstable binding of EMC3 and EMC6 with the substrate allows its dissociation from the EMC. Finally, the substrate encounters the lumenal EMC1, which has been proposed to act as a recruitment site for chaperones and co-factors involved in protein folding 15 .

Reconstructions of the human EMC revealed a 10 member complex with a tripartite configuration $^{15}$. Based exclusively in the ER, EMC1, EMC4, EMC7 and EMC10 form an Lshaped lumenal region, with EMC2 and EMC8 anchoring a basket shaped cytosolic region and a total of 12 TMDs making up the membrane spanning region in the ER ${ }^{15}$. Additional reports revealed that EMC1, EMC2, EMC3, EMC5 and EMC6 form the core while EMC4, EMC7 and EMC10 constitute peripheral components of the complex ${ }^{4,16}$.

The EMC may also play key roles in viral protein expression and replication by interacting with TMDs of viral proteins that are crucial for viral replication, contributing to their stabilization, folding and expression ${ }^{8}$. In human cells, the absence of the EMC caused a subset of transmembrane proteins to be inaccurately expressed and integrated into membranes, leading to a substantial reduction in infection rate of the viruses ${ }^{8}$. This paves way for the EMC to be explored as potential antiviral target. Furthermore, recent reports implicated individual EMC subunits in cardiovascular and neurodevelopmental diseases ${ }^{17}$.

Analyses by homology searching algorithms have deduced that the EMC was present in the last eukaryote common ancestor (LECA) ${ }^{1}$, making the study of EMCs an interesting pursuit apropos the evolution of ancestral ER proteins. Experimental studies on the EMC have so far been limited to the Opisthokonts ${ }^{18}$, i.e. they have remained unexplored in the eukaryotic 
supergroup of Excavata, which Trypanosoma belong to. Among protozoan parasites such as Giardia intestinalis and Trichomonas vaginalis, trypanosomes are the only organisms displaying a full set of (predicted) EMC subunits ${ }^{1}$. The conserved nature of the EMCs in conjunction with the position of $T$. brucei in the eukaryotic evolutionary tree provides a unique opportunity to study the role of the EMC in a highly diverged eukaryote that is essentially unrelated to Opisthokonts. This may help to reveal its ancestral function(s) in the ER and shed light on the root of its pleiotropy.

Trypanosoma brucei is a eukaryotic protozoan parasite that causes African sleeping sickness in humans and nagana in cattle. Trypanosomes are digenetic parasites and have the ability to metabolically as well as morphologically adapt to vastly different environments in their insect vector and mammalian hosts ${ }^{19,20}$. The parasites alternate between bloodstream forms that rely on glycolysis for ATP production while insect (procyclic) forms generate energy primarily by amino acid oxidation ${ }^{21,22}$. Several conserved and important biological pathways have initially been described in trypanosomes, such as glycosylphosphatidylinositol (GPI) anchoring of proteins ${ }^{23}$, RNA editing ${ }^{24}$, antigenic variation ${ }^{25}$ and trans-splicing ${ }^{26}$. Unlike mammals and yeast, trypanosomes have a single continuous mitochondrion harboring the mitochondrial genome or kinetoplast $\mathrm{DNA}^{27,28}$; its replication is in concert with the nuclear cell cycle, a peculiarity rarely seen in other eukaryotes ${ }^{29,30} . T$. brucei parasites are also capable of de novo lipid synthesis and contain phospholipid compositions similar to other eukaryotes $^{31,32}$, with phosphatidylcholine (PC) and phosphatidylethanolamine (PE) representing the two most abundant phospholipid classes ${ }^{31,33}$. In T. brucei bloodstream and procyclic forms, PC and PE are synthesized exclusively by the CDP-choline and CDPethanolamine, respectively, branches of the Kennedy pathway ${ }^{31,34-36}$.

In this study, we use biochemical and microscopic imaging approaches to identify the EMC in T. brucei (TbEMC) and to study possible functions of individual TbEMC subunits. 
Our work reveals that the TbEMC is composed of 9 subunits that are present in a complex localizing to the mitochondrial-ER interface. Interestingly, depletion of individual TbEMC subunits lead to selective disruption of de novo formation of PC or PE, re-emphasizing the role of the EMC in phospholipid synthesis. Thus, the involvement of the EMC in phospholipid metabolism may represent an ancestral function. 


\section{Results}

\section{BLAST searches reveal homologs of human and yeast EMC subunits in T. brucei.}

By conducting pBLAST searches using the amino acid sequences of the individual members of the human and $S$. cerevisiae EMCs as queries, we identified 9 putative $T$. brucei EMC (TbEMC) proteins, with similarities of $20-40 \%$ with the corresponding yeast and human homologs (Fig. 1). This is in agreement with a previous phylogenetic analysis of EMC distribution in eukaryotes ${ }^{1}$. All TbEMC subunits except TbEMC2 and TbEMC 8 are predicted to contain 1-3 TMDs, similar to their human and S. cerevisiae EMC homologs ${ }^{15,37}$ (Fig. 1), and TbEMC1, TbEMC3, TbEMC5, TbEMC7 and TbEMC10 are predicted to contain two signal peptides each, but according to iPSORT ${ }^{38}$ are not targeted to the mitochondrion.

\section{Depletion of single TbEMC subunits inhibits $T$. brucei growth in culture}

To study the essentiality of individual TbEMC subunits for growth of T. brucei procyclic forms, we attempted to generate null mutants using CRISPR/CRISPR-associated protein 9mediated gene deletion ${ }^{39}$. We successfully generated TbEMC7-knockout (TbEMC7-KO) and TbEMC10-KO cell lines and found that parasite growth was decreased after deletion of TbEMC7 (with an increase in cell doubling time from $9.0 \mathrm{~h}$ in parental to $12.5 \mathrm{~h}$ in TbEMC7KO parasites) (Fig. 2A), but not after deletion of TbEMC10 (Fig. 2B). Deletion of the respective genes was verified by PCR (Fig. S1). Attempts to delete the genes encoding TbEMC1, TbEMC3, or TbEMC8 were not successful, which prompted us to down-regulate their expression using tetracycline-inducible RNA interference (RNAi). The results revealed that growth of parasites cultured in the presence of tetracycline, to ablate TbEMC expression, was arrested after approximately 2 days, demonstrating that expression of TbEMC1, TbEMC3 
and TbEMC8 is essential for normal growth of T. brucei procyclic forms in culture (Fig. 2CE; Fig. S2).

\section{Localization and organization of the TbEMC}

To study their subcellular localization, we in situ-tagged all 9 TbEMC proteins in separate cell lines using HA (TbEMC1-4) or cMyc (TbEMC5-10) as tags. All TbEMCs were tagged C-terminally except TbEMC6, which was N-terminally tagged; C-terminal tagging of TbEMC6 was repeatedly unsuccessful. Analysis by SDS-PAGE and immunoblotting showed that all TbEMC proteins were expressed and migrated according to their predicted molecular masses (Fig. 3A, B). TbEMC8 and TbEMC10 consistently appeared as double bands. Analysis by immunofluorescence microscopy revealed that most TbEMC proteins co-localized with the ER lumenal marker protein $\mathrm{BiP}^{40}$, except TbEMC6 and TbEMC10, which exhibited preferential co-localization with ATOM40, the mitochondrial outer membrane protein- and tRNA-translocase in T. brucei ${ }^{41,42}$ (Fig. 4).

Most EMC subunits in mammalian cells and S. cerevisiae interact with each other ${ }^{7,43}$. To identify possible interactions between individual TbEMCs, we generated T. brucei procyclic forms co-expressing HA- and cMyc-tagged TbEMC proteins. We expressed TbEMC5-cMyc in tandem with TbEMC1-HA, TbEMC2-HA, TbEMC3-HA or TbEMC4-HA, and TbEMC3HA in tandem with TbEMC6-cMyc, TbEMC7-cMyc, TbEMC8-cMyc or TbEMC10-cMyc, with each combination in separate parasites. Co-immunoprecipitation experiments were performed to probe for interactions between individual TbEMC proteins in the double-tagged parasites. Using TbEMC5-cMyc as bait, we were able to pull-down TbEMC1-HA, TbEMC2HA, TbEMC3-HA and TbEMC4-HA (Fig. 5A-D). In reciprocal co-immunoprecipitation experiments, TbEMC5-cMyc was pulled-down only when using TbEMC2-HA as bait (Fig. 5A-D). In addition, when TbEMC3-HA was used as bait, TbEMC7-cMyc, TbEMC8-cMyc and 
TbEMC10-cMyc co-precipitated. TbEMC3-HA was faintly detected only in a pull-down using TbEMC8-cMyc as bait (Fig. 5E-G).

Furthermore, using immunofluorescence microscopy, we found high degrees of colocalization between TbEMC5-cMyc and TbEMC1-HA, TbEMC2-HA, TbEMC3-HA and TbEMC4-HA, while TbEMC3-HA and TbEMC8-cMyc showed a weaker co-localization (Fig. 5I). No co-localization was seen between TbEMC3-HA and TbEMC10-cMyc (Fig. 5I).

\section{TbEMC subunits form a high molecular mass complex}

The yeast and mammalian EMC subunits form a single high molecular mass complex ${ }^{2,7,16}$. To investigate if the individual TbEMC proteins are also members of a complex in T. brucei, we performed native polyacrylamide gel electrophoresis (native PAGE) using $T$. brucei procyclic forms expressing the different epitope-tagged TbEMCs. Our results show that all 9 TbEMC proteins migrated as a high molecular mass complex of approximately $480 \mathrm{kDa}$ after native PAGE analyses (Fig. 6A, B). An unspecific band at approximately $720 \mathrm{kDa}$ appeared in all samples, including untagged parental parasites, probed with the anti-HA antibody. Together with the TbEMC co-immunoprecipitation studies, these results demonstrate that the TbEMC subunits not only interact with each other but form a single high molecular mass complex.

\section{Depletion of TbEMC7 destabilizes the TbEMC and leads to release of TbEMC3 from the}

\section{complex}

To study if the depletion of an individual subunit affects the integrity and/or stability of the TbEMC, we expressed in situ-tagged TbEMC3-cMyc in TbEMC7-KO parasites. Analysis by native PAGE revealed that in the absence of TbEMC7, TbEMC3-cMyc was not detectable (Fig. 7A), whereas in control parasites expressing TbEMC3-cMyc, the high molecular mass 
band at $480 \mathrm{kDa}$ was clearly visible (compare also with Fig. 6A, showing parasites expressing TbEMC3-HA). Analysis of the same samples by immunoblotting under denaturing conditions demonstrated that TbEMC3-cMyc was expressed in the TbEMC7-KO background and that its expression level was similar to that in control parasites expressing TbEMC3-cMyc (Fig. 7B), ruling out the possibility that TbEMC3-cMyc itself is unstable in parasites lacking TbEMC7. These results suggest that in the absence of TbEMC7, TbEMC3-cMyc no longer associates with the complex and/or that the complex is destabilized. To distinguish between these possibilities, we expressed TbEMC8-cMyc in the TbEMC7-KO background and analyzed the presence of the TbEMC by native PAGE. The results show that in TbEMC7-KO parasites the $480 \mathrm{kDa}$ band was reduced in intensity compared to control parasites (Fig. 7C), indicating that in the absence of the TbEMC7 subunit, the TbEMC is not properly formed or is destabilized. In line with these results, immunofluorescence microscopy analysis of TbEMC3-cMyc in TbEMC7-KO parasites revealed a diffuse staining pattern as compared to the punctate staining of TbEMC3-cMyc in control parasites (Fig. 7E), indicating that the TbEMC is not properly assembled. In contrast, in TbEMC10-KO parasites, the migration of TbEMC3-cMyc on native PAGE was unchanged compared to control cells (Fig. S3), indicating that the loss of TbEMC10 does not affect the integrity of the TbEMC.

\section{The TbEMC localizes to the mitochondrial-ER interface}

The EMC shows a considerable overlap with the ER and mitochondrion in T. brucei, and it is not unreasonable to assume that TbEMC clusters to the mitochondrial-ER interface, with the majority of the TbEMCs localizing to the ER and TbEMC6 and TbEMC10 interacting with the mitochondrial outer membrane (MOM). This would be in agreement with a previous study that implicates the EMC as an ER-mitochondrial tether in S. cerevisiae ${ }^{7}$. As conventional immunofluorescence microscopy techniques could not appreciably resolve the MOM and 
adjacent ER tubules, we wondered if the TbEMC could be traced biochemically to the mitochondrial ER-interface, in a separable membrane fraction termed mitochondria-associated ER membranes ${ }^{44}$. Established markers of these ER-subdomains associated with mitochondria are the phosphatidylserine synthases PSS1 and PSS2 ${ }^{45,46}$. To establish the sub-organellar localization of PSS2 in T. brucei, we generated a cell line expressing TbPSS2 (Tb927.7.3760) containing a C-terminal cMyc-tag from an ectopic locus under the control of tetracycline and executed a well-established cell fractionation protocol to generate highly purified mitochondrial vesicles (Fig. $8 \mathrm{~A})^{47}$. The resultant fractions were analyzed by immunoblotting and probed for cMyc and the MOM marker ATOM40.

Our results show that while the majority of TbPSS2-cMyc is present in a fraction containing microsomal ER vesicles, a significant amount also co-fractionated with two mitochondrial fractions (Fig. 8B). Additionally, when we isolated the MOM from the purified mitochondria fraction (employing a previously established procedure ${ }^{48}$ ), TbPSS2-cMyc and ATOM40 were equally enriched in both the MOM and the fraction containing both MOM and ER proteins (MOM/ER fraction) (Fig. 8C). Thus, a fraction of TbPSS2-cMyc — despite being an ER resident protein ${ }^{49}$ — stably associated and co-fractionated with the MOM. We next asked if the TbEMC subunits would fractionate in similar fashion as TbPSS2-cMyc, indicative of a common localization at the mitochondrial ER-interface. To investigate this, we re-analyzed the data from our previous study in which the above-mentioned fractions (Fig. 8A) had been subjected to mass spectrometry analyses to obtain protein abundance profiles ${ }^{48}$. We were able to confirm that all TbEMCs, except TbEMC6, were present in the individual fractions, with the protein abundance profiles shown in Fig. 8D, including the one for TbPSS2. Interestingly, we found that the protein abundance profiles of the TbEMCs closely matched that of TbPSS2, with maximal intensity in the MOM/ER fraction (Fig. 8D). These results are in good agreement with the immunoblot analyses shown in Fig. 8C. 
Finally, to confirm co-localization of the TbEMCs with TbPSS2 by immunofluorescence microscopy, we generated a cell line co-expressing TbEMC3-HA and TbPSS2-cMyc. Our results show that TbEMC3-HA strongly co-localized with TbPSS2-cMyc (Fig. 8E). Together, these data indicate that the TbEMC proteins and TbPSS2 localize to the same sub-organellar structure, which we refer to here as the mitochondrial ER-interface.

\section{Depletion of individual TbEMC proteins inhibits de novo phospholipid synthesis}

To determine if the TbEMC may play a role in the de novo synthesis of phospholipids in T. brucei, we analyzed incorporation of $\left[{ }^{3} \mathrm{H}\right]$-choline and $\left[{ }^{3} \mathrm{H}\right]$-ethanolamine into $\mathrm{PC}$ and $\mathrm{PE}$, respectively, in T. brucei procyclic forms after depletion of individual TbEMC proteins. The labeled precursors were added after $48 \mathrm{~h}$ of RNAi, i.e. before parasite growth was affected by down-regulation of the individual TbEMC proteins (see Fig. 2C-E), and incubated for $4 \mathrm{~h}$ to allow uptake and metabolism into phospholipids via the Kennedy pathway ${ }^{36}$. Formation of $\left[{ }^{3} \mathrm{H}\right]$-PI using [ $\left.{ }^{3} \mathrm{H}\right]-m y o-i n o s i t o l$ as precursor was used as internal labeling control. Lipids were separated by thin layer chromatography (TLC) and incorporation of radioactivity into different phospholipid classes was quantified by radioisotope scanning; a representative TLC analysis is shown in Fig. S4. We found that depletion of TbEMC1 or TbEMC3 decreased de novo synthesis of $\left[{ }^{3} \mathrm{H}\right]-\mathrm{PC}$ by $50-60 \%$ compared to control parasites (Fig. 9A, C). In contrast, downregulation of neither of these two TbEMCs had an effect on the synthesis of [ $\left.{ }^{3} \mathrm{H}\right]-\mathrm{PE}$ (Fig. 9B, D). Unexpectedly, the opposite effect was observed after depletion of TbEMC8. RNAi against TbEMC 8 decreased de novo synthesis of $\left[{ }^{3} \mathrm{H}\right]-\mathrm{PE}$ by $>60 \%$ compared to control parasites, while no effect was seen for $\left[{ }^{3} \mathrm{H}\right]$-PC formation (Fig. 9E, F). No major differences in $\left[{ }^{3} \mathrm{H}\right]-\mathrm{PC}$ or $\left[{ }^{3} \mathrm{H}\right]-\mathrm{PE}$ synthesis was seen in TbEMC7-KO (Fig. 9G, H) and TbEMC10-KO parasites (Fig. 9I, J). Together these results show that in T. brucei procyclic forms depletion of individual 
bioRxiv preprint doi: https://doi.org/10.1101/2021.06.17.448810; this version posted June 17, 2021. The copyright holder for this preprint (which was not certified by peer review) is the author/funder, who has granted bioRxiv a license to display the preprint in perpetuity. It is made available under aCC-BY 4.0 International license.

TbEMC proteins results in disturbance of select pathways for de novo synthesis of the major phospholipid classes. 


\section{Discussion}

The EMC has been implicated in many cellular processes, including inter-organellar lipid exchange ${ }^{7,10}$. Although lipidomic analyses have revealed that the loss of EMC3 leads to perturbations in cellular lipid levels in murine cells ${ }^{10}$, involvement of individual EMC subunits in the intricate pathways of lipid biosynthesis has not been studied before in detail. Here we demonstrate that RNAi-mediated ablation of TbEMC1, TbEMC3 or TbEMC8 expression inhibited production of PC or PE, the major phospholipid classes in T. brucei procyclic forms. PC and PE in T. brucei parasites are synthesized exclusively by the CDP-choline and CDPethanolamine, respectively, branches of the Kennedy pathway ${ }^{32}$. Both branches are essential for parasite survival in culture. The first two reactions of either branch, catalyzed by the respective kinases and phosphotransferases, occur in the cytosol. In contrast, the final reactions are mediated by the membrane-bound enzymes TbCEPT and TbEPT, respectively, in the ER. Interestingly, the two enzymes localize to different sub-compartments of the ER, with TbCEPT being present exclusively in the perinuclear ER and TbEPT localizing mostly to the bulk ER, but also with a fractional overlap with the perinuclear $\mathrm{ER}^{36}$. Since both enzymes have multiple TMDs, it is conceivable that the expression and/or the membrane insertion of TbCEPT and TbEPT may be affected following disruption of the TbEMC's attributed main function as membrane protein insertase. Our findings indicate that down-regulation of TbEMC1 or TbEMC3 resulted in inhibition of de novo production of PC, but had no effect on PE synthesis, while depletion of TbEMC8 had the opposite effect, i.e. inhibited de novo formation of PE without affecting PC synthesis. These results point to a more specific role of the respective TbEMC subunits in phospholipid synthesis. The localization of the TbEMC to the mitochondria-ER interface suggests that certain subunits may be involved in the transfer of phospholipid precursors or intermediates between these organelles. Alternatively, select subunits of the TbEMC may mediate the biogenesis of proteins involved in the transport of PC 
and PE, such as phospholipid exchange proteins or transporters, between (sub-compartments of) the ER and the mitochondrion. Whole cell proteomic analyses may reveal such possible links in future studies.

Recent work has shown that the presence of a hydrophilic vestibule or substrate binding pocket is central to the insertion mechanism of the EMC complex ${ }^{15,37}$. Simulations on the $S$. cerevisiae EMC structure have revealed that perturbations in the cytoplasmic leaflet of the ER bilayer facilitates flooding of the substrate binding pocket with water, promoting a hydrophilic environment instrumental to insertion ${ }^{50}$. Since PC and PE are major structural components of the ER membrane, it is tempting to speculate that the role of the EMC in lipid biosynthesis is tied to the creation of a microenvironment conducive to TMD insertion, and thus EMC function.

The EMC in mammalian and $S$. cerevisiae cells localizes exclusively to the ER. In analogy, most TbEMC subunits also localized to the ER and formed a complex in T. brucei. However, unexpectedly, TbEMC6 and TbEMC10 exhibited mitochondrial localization. This together with our biochemical data showing that the TbEMC proteins and TbPSS2 cofractionate in a distinct sub-organellar membrane fraction, localize the TbEMC to the mitochondria-ER interface. A similar localization of the EMC in close proximity to both organelles has been proposed in $S$. cerevisiae, where it was postulated to act as an ERmitochondrial tether?

Interestingly, unlike in mammalian cells where EMC7 is considered a peripheral component of the complex ${ }^{16}$, we identified TbEMC7 as a crucial subunit integral to the stability of the TbEMC. In its absence, the TbEMC is not properly formed or is destabilized, resulting in at least the release of TbEMC3 from the complex. TbEMC7-KO parasites continue to synthesize PC, albeit less efficiently, and are viable. In contrast, RNAi-mediated depletion of TbEMC3 results in growth arrest. Together, these results indicate that TbEMC3 is able to, at 
least partially, maintain its role in PC synthesis even after release from the TbEMC. Alternatively, TbEMC3 may retain its function in PC synthesis via transient association with the TbEMC in TbEMC7-KO parasites.

Finally, human EMC10 was reported to juggle many roles, by acting as an angiogenetic growth factor ${ }^{51}$, an inhibitor of neuronal maturation involved in schizophrenia-related phenotypes $^{52}$, and as transcription modulator in malignant glioma cells ${ }^{53}$. In light of these pleotropic functions in human cells, and the lack of effects of TbEMC10 depletion on parasite growth, phospholipid synthesis, or stability of the TbEMC, it would not be surprising if TbEMC10 also exhibits moonlighting functions in T. brucei. 


\section{Materials and Methods}

\section{Reagents}

All reagents were of analytical grade and purchased from Merck-Sigma Aldrich (Darmstadt, Germany) or MilliporeSigma (Burlington, MA, USA) unless otherwise stated. Kits for plasmid DNA extractions, PCR purifications, vector dephosphorylation and ligation reactions were purchased from Promega (Wallisellen, Switzerland). All sequencing was done by Microsynth AG (Balgach, Switzerland).

\section{Cell culture and knockdown trypanosome cell lines}

Procyclic form trypanosomes were cultured at $27{ }^{\circ} \mathrm{C}$ in SDM7954 supplemented with $10 \%$ heat inactived fetal bovine serum (Thermo Fisher Scientific, Reinach, Switzerland) and a mixture of hemin and folic acid $(160 \mu \mathrm{M}$ hemin $+90 \mu \mathrm{M}$ folic acid). Procyclic form (SmOxP9) trypanosomes expressing tagged TbEMCs were cultured in the presence of hygromycin (25 $\mu \mathrm{g} / \mathrm{ml})$, G418 $(15 \mu \mathrm{g} / \mathrm{ml})$, puromycin $(2 \mu \mathrm{g} / \mathrm{ml})$, blasticidin $(5 \mu \mathrm{g} / \mathrm{ml})$. TbEMC-KO parasites were cultured in the presence of hygromycin $(25 \mu \mathrm{g} / \mathrm{ml}), \mathrm{G} 418(15 \mu \mathrm{g} / \mathrm{ml})$, puromycin (2 $\mu \mathrm{g} / \mathrm{ml})$ and blasticidin $(5 \mu \mathrm{g} / \mathrm{ml})$, and TbEMC-KO parasites expressing in-situ tagged proteins were cultured in the presence of an additional $0.2 \mu \mathrm{g} / \mathrm{ml}$ of phleomycin. T. brucei strain 29$13^{55}$ was used to generate RNAi cell lines against TbEMC1, TbEMC3 and TbEMC8, and cultured in the presence of hygromycin $(25 \mu \mathrm{g} / \mathrm{ml})$ and $\mathrm{G} 418(15 \mu \mathrm{g} / \mathrm{ml})$, and puromycin $(2$ $\mu \mathrm{g} / \mathrm{ml})$, blasticidin $(5 \mu \mathrm{g} / \mathrm{ml})$ and phleomycin $(0.2 \mu \mathrm{g} / \mathrm{ml})$ in TbEMC1 RNAi, TbEMC3 RNAi and TbEMC8 RNAi, respectively, parasites. For induction of RNAi tetracycline $(1 \mu \mathrm{g} / \mathrm{ml})$ was added. The RNAi constructs were based on a derivate of the stem-loop vector pLEW $100^{55}$ using the following inserts for the RNAi construct: TbEMC1 (Tb927.4.590) ORF nt 996-1246, TbEMC3 (Tb927.10.4760) 3' UTR nt 13-165 and TbEMC8 (Tb927.8.1570) ORF nt 2084- 
2501. Transfection and selection of clones of the above-mentioned TbEMC knock-downs were performed as described previously ${ }^{56}$ and RNAi efficiency was verified by mRNA Northern blotting.

\section{Generation of TbEMC-KO parasites using clustered regularly interspaced short palindromic repeats (CRISPR)/CRISPR associated protein 9}

CRISPR/Cas9 was employed to create knockouts of individual TbEMCs, as described previously ${ }^{39}$. Briefly, procyclic form (SmOxP9) trypanosomes were used to create knockouts by replacing the TbEMC gene on both alleles with 2 antibiotic resistance cassettes by transfection. The 2 antibiotic resistance cassettes flanked by homology sequences of $30 \mathrm{nt}$ were amplified by PCR reactions using pPOTv6 plasmids ${ }^{57}$ with hygromycin and geneticin resistance cassettes. Both the antibiotic cassettes as well as short guide RNA templates consisting of a $\mathrm{T} 7$ promoter sequence, a Cas9 binding site, and $20 \mathrm{nt}$ long target sequence were amplified as described previously ${ }^{39}$. Primers were generated by www.leishgedit.net (Table S5) and all PCR reactions were performed using Expand High Fidelity PCR System (Roche, Basel, Switzerland), pooled and purified using PCR purification kits. Transfection was performed using a 4D-Nucleofector system, with the FI-115 program (Lonza, Basel, Switzerland). The transfected culture was distributed to 24 -well plates after a $2 \mathrm{~h}$ recovery and $22.5 \mu \mathrm{g} / \mathrm{ml}$ geneticin and $37.5 \mu \mathrm{g} / \mathrm{ml}$ hygromycin (selective antibiotics) were added after $24 \mathrm{~h}$.

TbEMC-KO genotypes were verified by PCR analysis of genomic DNA isolated from parental and knockout cell lines. All primer pairs and PCRs conducted for TbEMC knockout verification are listed in Fig. S1.

\section{In-situ tagging of TbEMCs}


TbEMCs were tagged in SmOxP9 as well as TbEMC-KO parasites using CRISPR/Cas9 technique as described above. Briefly, resistance cassette consisting of a cMyc tag sequence and a blasticidin/hygromycin/phleomycin resistance gene and homology sequences was inserted in TbEMC genes by homologous recombination. Corresponding guide RNA templates were also generated. All primers were generated by www.leishgedit.net (Table S6). The PCR products were pooled, purified, and transfected as described above. 24 hours after distribution into 24-well plates, selection antibiotics were added. TbEMC protein expression in the parasites was analyzed by SDS-PAGE and immunoblotting. TbPSS2 cell line has been described previously ${ }^{49}$.

\section{Whole cell lysates, crude membrane preparations and immunoprecipitations}

For whole cell lysate preparations, $10^{7}$ trypanosomes were harvested, and the resultant pellet was washed with Tris-buffered saline (TBS, $10 \mathrm{mM}$ Tris-HCl, pH 7.5, $144 \mathrm{mM} \mathrm{NaCl}$ ) and resuspended in sample buffer $\left(2.5 \%\right.$ SDS). The sample was then incubated at $50{ }^{\circ} \mathrm{C}$ for 5 $\min$.

For crude membrane preparations, a digitonin extraction was performed. Trypanosomes $\left(10^{8}\right.$ cells) were harvested by centrifugation, washed in TBS and resuspended in $0.5 \mathrm{ml}$ buffer (20 mM Tris-HCl, pH 7.5, 600 mM sorbitol, 2 mM EDTA) containing 0.05\% (w/v) digitonin. The suspension was centrifuged at $6000 \mathrm{x}$ g. Subsequently, $100 \mu$ l extraction buffer $(20 \mathrm{mM}$ Tris- $\mathrm{HCl}, \mathrm{pH} 7.2,15 \mathrm{mM} \mathrm{NaH}_{2} \mathrm{PO}_{4}, 0.6 \mathrm{M}$ sorbitol) containing $1.5 \%(\mathrm{w} / \mathrm{v})$ digitonin was added to the pellet. The sample was incubated on ice for $15 \mathrm{~min}$ and membranes were pelleted by centrifugation at $16^{\prime} 000 \mathrm{x} \mathrm{g}$ in a tabletop centrifuge $\left(15 \mathrm{~min}, 4^{\circ} \mathrm{C}\right)$. The resultant pellet was used for further analysis.

For immunoprecipitations, whole cell lysates were solubilized in $1 \mathrm{ml}$ lysis buffer (10 mM Tris- HCl, pH 7.4, 150 mM NaCl, 1 mM EDTA, 1\% Triton X-100, 0.5\% NP-40, protease 
inhibitors) and incubated on ice for $30 \mathrm{~min}$. After centrifugation at $16^{\prime} 000 \mathrm{x} \mathrm{g}\left(15 \mathrm{~min}, 4^{\circ} \mathrm{C}\right)$ the lysate (input) was incubated with anti-HA or anti-cMyc antibodies (9E10; Santa Cruz Biotechnology, Dallas, TX, USA / 16B12 Enzo Life Sciences, Farmingdale NY, USA; dilution 1:40) in combination with $50 \mu \mathrm{l}$ Protein G Dynabeads (Thermo Fischer Scientific) for $16 \mathrm{~h}$. Bound proteins were eluted by addition of sample buffer and heating at $50{ }^{\circ} \mathrm{C}$ for $5 \mathrm{~min}$. The proteins were analyzed by SDS-PAGE and immunoblotting.

\section{PAGE}

Proteins were denatured by SDS and run on $10 \%$ polyacrylamide gels as described previously $^{58}$. For native PAGE, samples were prepared and loaded onto $4-12 \%$ gradient gels as described elsewhere ${ }^{59}$. The proteins were then transferred onto PVDF membranes (Thermo Fisher Scientific) using a semidry protein blotting system (Bio-Rad, Hercules, CA, USA) for 75 min at a maximal current of $2.5 \mathrm{~mA} \mathrm{~cm}^{-2}$ gel area. The membranes were then blocked for $1 \mathrm{~h}$ in TBS, containing 5\%(w/v) milk powder, and proteins of interest were identified using specific primary and horseradish peroxidase-conjugated secondary antibodies. Protein-specific antibodies were visualized using SuperSignal ${ }^{\mathrm{TM}}$ West Pico PLUS Chemiluminescent Substrate (Thermo Fisher Scientific). The following antibodies were diluted in TBS, containing 5\% milk: mouse monoclonal anti-cMyc (9E10; Santa Cruz Biotechnology; dilution 1:1000); mouse monoclonal anti-HA (HA11; Enzo Life Sciences; dilution 1:3000); rabbit anti-AAC1 (kindly provided by Alena P. Ziková, University of South Bohemia, Ceske Budejovice, Czech Republic; dilution 1:20000); HRP-conjugated anti-rabbit and anti-mouse antibody (DAKOAgilent, Basel, Switzerland; dilution 1:1000 and 1:5000, respectively).

\section{Immunofluorescence microscopy}


For immunofluorescence staining of trypanosomes, $10^{6}$ cells were harvested by centrifugation, resuspended in ice cold PBS (phosphate-buffered saline; $137 \mathrm{mM} \mathrm{NaCl}, 2.7$ $\mathrm{mM} \mathrm{KCl}, 10 \mathrm{mM} \mathrm{Na}_{2} \mathrm{HPO}_{4}, 2 \mathrm{mM} \mathrm{KH}_{2} \mathrm{PO}_{4}, \mathrm{pH}$ 7.4), allowed to adhere onto glass slides (Thermo Fisher Scientific) and fixed with 4\% (w/v) paraformaldehyde for $10 \mathrm{~min}$. The cells were then washed with PBS and permeabilized with $0.2 \%(\mathrm{w} / \mathrm{v})$ Triton X-100 for $5 \mathrm{~min}$. After blocking in PBS containing 2\% (w/v) BSA, for $30 \mathrm{~min}$, parasites were incubated with antibodies diluted in blocking solution for $45 \mathrm{~min}$. The following primary antibodies were used: rabbit anti-cMyc (LubioSciences, Zurich, Switzerland; dilution 1:200); mouse monoclonal anti-cMyc (9E10, Santa Cruz Biotechnology; dilution 1:200), mouse anti-HA (16B12; Enzo Life Sciences; dilution 1:200), rabbit anti-ATOM40 (dilution 1:1000), rabbit anti-BiP (kindly provided by J. Bangs, University of Buffalo, NY, USA; dilution 1:20,000) . After 3 washes in PBS for 5 min each, the cells were incubated with the corresponding secondary fluorophoreconjugated antibodies Alexa Fluor goat anti-mouse 488 and goat anti-rabbit 594 (Thermo Fisher Scientific; dilution 1:1000) in blocking solution for $45 \mathrm{~min}$. Cells were washed, air dried and mounted with Vectashield (Vector Laboratories, Burlingame, CA, USA) containing 4',6diamidino-2-phenylindole (DAPI).

Fluorescence microscopy was performed with a Leica DM 16000 B inverted microscope using a 60x oil objective and images were acquired using Leica DFC360 FX camera. Image deconvolution and processing was performed using Leica LAS X and Fiji software (NIH).

\section{$\left[{ }^{3} \mathrm{H}\right]$-glycerol labeling and lipid analysis}

Trypanosomes $\left(10^{8}\right.$ cells $)$ were labeled with $50 \mu \mathrm{Ci}\left[{ }^{3} \mathrm{H}\right]$-ethanolamine or $50 \mu \mathrm{Ci}\left[{ }^{3} \mathrm{H}\right]$ choline chloride in combination with $20 \mu \mathrm{Ci}\left[{ }^{3} \mathrm{H}\right]$-inositol (Anawa, Kloten, Switzerland) for 4 $\mathrm{h}$ before being harvested by centrifugation and washed twice with TBS. The radiolabeled lipids were extracted according to Bligh and Dyer ${ }^{60}$, spotted onto silica gel plates (Merck, Zug, 
Switzerland) and separated by one dimensional TLC using a solvent system consisting of chloroform: methanol: acetic acid (65: 25: 8, by vol) to track PE formation and chloroform: methanol: acetic acid: water (50:30:8: 4, by vol) to track PC formation. The plates were dried, and radioactivity was detected using a radioisotope scanner (Berthold Technologies, Bad Wildbad, Germany). Data processing was done using the Rita Control software provided by the manufacturer.

\section{Preparation of mitochondrial vesicles, mitochondrial outer membrane (OM) and OM/ER}

\section{fractions}

The detailed procedure for the purification of mitochondrial vesicles from a variety of different cell amounts is detailed eslewhere ${ }^{47}$. Briefly, trypanosomes were lysed at isotonic conditions using nitrogen cavitation, and following differential centrifugation, mitochondrial vesicles were purified from Nycodenz step-gradients subjected to density gradient centrifugation. Mitochondrial OM was further purified from these mitochondrial vesicles according to a previously described protocol ${ }^{48}$.

\section{Acknowledgments}

The work was funded by Swiss National Science Foundation grants 169355 (to PB) and 175563 (to AS) and in part by the NCCR "RNA \& Disease" funded by the Swiss National Science Foundation (to AS). AI thanks S. Iyer and P.G. Balan for their support. PB thanks R. Plant, M. Bütikofer and L. Apitzsch for their support. 


\section{References}

1. Wideman, J. G. The ubiquitous and ancient ER membrane protein complex (EMC): tether or not? F1000Research (2015) doi:10.12688/f1000research.6944.2.

2. Jonikas, M. C. et al. Comprehensive characterization of genes required for protein folding in the endoplasmic reticulum. Science (80-. ). (2009) doi:10.1126/science.1167983.

3. Louie, R. J. et al. A yeast phenomic model for the gene interaction network modulating CFTR- $\Delta$ F508 protein biogenesis. Genome Med. 4, (2012).

4. Chitwood, P. J. \& Hegde, R. S. The Role of EMC during Membrane Protein Biogenesis. Trends in Cell Biology (2019) doi:10.1016/j.tcb.2019.01.007.

5. Miller-Vedam, L. E. et al. Structural and mechanistic basis of the EMC-dependent biogenesis of distinct transmembrane clients. Elife 9, (2020).

6. Volkmar, N. \& Christianson, J. C. Squaring the EMC - How promoting membrane protein biogenesis impacts cellular functions and organismal homeostasis. Journal of Cell Science vol. 133 (2020).

7. Lahiri, S. et al. A Conserved Endoplasmic Reticulum Membrane Protein Complex (EMC) Facilitates Phospholipid Transfer from the ER to Mitochondria. PLoS Biol. (2014) doi:10.1371/journal.pbio.1001969.

8. Ngo, A. M. et al. The ER membrane protein complex is required to ensure correct topology and stable expression of flavivirus Polyproteins. Elife (2019) doi:10.7554/eLife.48469.

9. Shurtleff, M. J. et al. The ER membrane protein complex interacts cotranslationally to enable biogenesis of multipass membrane proteins. Elife (2018) doi:10.7554/eLife.37018. 
10. Tang, X. et al. EMC3 coordinates surfactant protein and lipid homeostasis required for respiration. J. Clin. Invest. (2017) doi:10.1172/JCI94152.

11. Satoh, T. \& Satoh, A. K. EMC is essential for biosynthesis of rhodopsin and other multipass membrane proteins in Drosophila photoreceptors. Seikagaku. The Journal of Japanese Biochemical Society (2015).

12. Richard, M., Boulin, T., Robert, V. J. P., Richmond, J. E. \& Bessereau, J. L. Biosynthesis of ionotropic acetylcholine receptors requires the evolutionarily conserved ER membrane complex. Proc. Natl. Acad. Sci. U. S. A. (2013) doi:10.1073/pnas.1216154110.

13. Guna, A., Volkmar, N., Christianson, J. C. \& Hegde, R. S. The ER membrane protein complex is a transmembrane domain insertase. Science (80-. ). (2018) doi:10.1126/science.aao3099.

14. Tian, S. et al. Proteomic Analysis Identifies Membrane Proteins Dependent on the ER Membrane Protein Complex. Cell Rep. 28, 2517-2526.e5 (2019).

15. Pleiner, T. et al. Structural basis for membrane insertion by the human ER membrane protein complex. Science (80-. ). 369, (2020).

16. Volkmar, N. et al. The ER membrane protein complex promotes biogenesis of sterolrelated enzymes maintaining cholesterol homeostasis. J. Cell Sci. (2019) doi:10.1242/jcs.223453.

17. Marquez, J. et al. Disrupted ER membrane protein complex-mediated topogenesis drives congenital neural crest defects. J. Clin. Invest. (2020) doi:10.1172/JCI129308.

18. Simpson, A. G. B. \& Roger, A. J. The real 'kingdoms' of eukaryotes. Current biology : $C B$ vol. 14 (2004).

19. Fenn, K. \& Matthews, K. R. The cell biology of Trypanosoma brucei differentiation. Current Opinion in Microbiology vol. 10 (2007). 
20. Sharma, R. et al. The heart of darkness: growth and form of Trypanosoma brucei in the tsetse fly. Trends in Parasitology vol. 25 (2009).

21. Michels, P. A. M., Bringaud, F., Herman, M. \& Hannaert, V. Metabolic functions of glycosomes in trypanosomatids. Biochimica et Biophysica Acta - Molecular Cell Research vol. 1763 (2006).

22. Vickerman, K. Developmental cycles and biology of pathogenic trypanosomes. $\mathrm{Br}$. Med. Bull. 41, (1985).

23. Ferguson, M. A. J., Homans, S. W., Dwek, R. A. \& Rademacher, T. W. Glycosylphosphatidylinositol moiety that anchors Trypanosoma brucei variant surface glycoprotein to the membrane. Science (80-. ). 239, (1988).

24. Blum, B., Bakalara, N. \& Simpson, L. A model for RNA editing in kinetoplastid mitochondria: RNA molecules transcribed from maxicircle DNA provide the edited information. Cell 60, (1990).

25. Cross, G. A. M. Antigenic variation in trypanosomes: Secrets surface slowly. BioEssays 18, (1996).

26. Sutton, R. E. \& Boothroyd, J. C. Evidence for Trans splicing in trypanosomes. Cell 47, (1986).

27. Tyler, K. M., Matthews, K. R. \& Gull, K. Anisomorphic cell division by African trypanosomes. Protist 152, (2001).

28. Povelones, M. L. Beyond replication: division and segregation of mitochondrial DNA in kinetoplastids. Molecular and biochemical parasitology vol. 196 (2014).

29. Robinson, D. R. \& Gull, K. Basal body movements as a mechanism for mitochondrial genome segregation in the trypanosome cell cycle. Nature 352, (1991).

30. Schneider, A. \& Ochsenreiter, T. Failure is not an option - mitochondrial genome segregation in trypanosomes. J. Cell Sci. 131, (2018). 
31. Smith, T. K. \& Bütikofer, P. Lipid metabolism in Trypanosoma brucei. Molecular and Biochemical Parasitology vol. 172 (2010).

32. Ramakrishnan, S., Serricchio, M., Striepen, B. \& Bütikofer, P. Lipid synthesis in protozoan parasites: A comparison between kinetoplastids and apicomplexans. Progress in Lipid Research vol. 52 (2013).

33. Patnaik, P. K. et al. Molecular species analysis of phospholipids from Trypanosoma brucei bloodstream and procyclic forms. Mol. Biochem. Parasitol. 58, (1993).

34. KENNEDY, E. P. \& WEISS, S. B. The function of cytidine coenzymes in the biosynthesis of phospholipides. J. Biol. Chem. 222, (1956).

35. Signorell, A., Rauch, M., Jelk, J., Ferguson, M. A. J. \& Bütikofer, P. Phosphatidylethanolamine in Trypanosoma brucei is organized in two separate pools and is synthesized exclusively by the kennedy pathway. J. Biol. Chem. 283, (2008).

36. Farine, L., Niemann, M., Schneider, A. \& Bütikofer, P. Phosphatidylethanolamine and phosphatidylcholine biosynthesis by the Kennedy pathway occurs at different sites in Trypanosoma brucei. Sci. Rep. 5, (2015).

37. Bai, L., You, Q., Feng, X., Kovach, A. \& Li, H. Structure of the ER membrane complex, a transmembrane-domain insertase. Nature 584, (2020).

38. Bannai, H., Tamada, Y., Maruyama, O., Nakai, K. \& Miyano, S. Extensive feature detection of N-terminal protein sorting signals. Bioinformatics 18, (2002).

39. Beneke, T. et al. A CRISPR-Cas9 high-throughput genome editing toolkit for kinetoplastids: Supplementary information. Handb. Gener. IV Nucl. React. (2016) doi:10.1016/B978-0-08-100149-3.15007-4.

40. Bangs, J. D., Uyetake, L., Brickman, M. J., Balber, A. E. \& Boothroyd, J. C. Molecular cloning and cellular localization of a BiP homologue in trypanosoma brucei. Divergent ER retention signals in a lower eukaryote. J. Cell Sci. 105, (1993). 
41. Pusnik, M. et al. Mitochondrial preprotein translocase of trypanosomatids has a bacterial origin. Curr. Biol. 21, (2011).

42. Niemann, M. et al. tRNAs and proteins use the same import channel for translocation across the mitochondrial outer membrane of trypanosomes. Proc. Natl. Acad. Sci. U. S. A. 114, (2017).

43. Christianson, J. C. et al. Defining human ERAD networks through an integrative mapping strategy. Nat. Cell Biol. (2012) doi:10.1038/ncb2383.

44. Vance, J. E. Phospholipid synthesis in a membrane fraction associated with mitochondria. J. Biol. Chem. 265, (1990).

45. Stone, S. J. \& Vance, J. E. Phosphatidylserine synthase-1 and -2 are localized to mitochondria-associated membranes. J. Biol. Chem. 275, (2000).

46. Area-Gomez, E. et al. Upregulated function of mitochondria-associated ER membranes in Alzheimer disease. EMBO J. 31, (2012).

47. Niemann, M. \& Schneider, A. A Scalable Purification Method for Mitochondria from Trypanosoma brucei. in Methods in Molecular Biology vol. 2116 (2020).

48. Niemann, M. et al. Mitochondrial outer membrane proteome of trypanosoma brucei reveals novel factors required to maintain mitochondrial morphology. Mol. Cell. Proteomics 12, (2013).

49. Farine, L. et al. Phosphatidylserine synthase 2 and phosphatidylserine decarboxylase are essential for aminophospholipid synthesis in Trypanosoma brucei. Mol. Microbiol. 104, (2017).

50. Alvira, S., Corey, R. A., Collinson, I. \& Römisch, K. Membrane protein biogenesis by the EMC. EMBO J. 40, (2021).

51. Reboll, M. R. et al. EMC10 (Endoplasmic reticulum membrane protein complex subunit 10) is a bone marrow-derived angiogenic growth factor promoting tissue repair 
after myocardial infarction. Circulation 136, (2017).

52. Diamantopoulou, A. et al. Loss-of-function mutation in Mirta22/Emc10 rescues specific schizophrenia-related phenotypes in a mouse model of the 22q11.2 deletion. Proc. Natl. Acad. Sci. U. S. A. 114, (2017).

53. Junes-Gill, K. S. et al. hHSS1: A novel secreted factor and suppressor of glioma growth located at chromosome 19q13.33. J. Neurooncol. 102, (2011).

54. Brun, R. \& Schonenberger, M. Cultivation and in vitro cloning of procyclic culture forms of Trypanosoma brucei in a semi-defined medium. Short communication. Acta Trop. 36, (1979).

55. Wirtz, E., Leal, S., Ochatt, C. \& Cross, G. A. M. A tightly regulated inducible expression system for conditional gene knock-outs and dominant-negative genetics in Trypanosoma brucei. Mol. Biochem. Parasitol. (1999) doi:10.1016/S01666851(99)00002-X.

56. Waldman, A. Genetic Recombination: Reviews and Protocols. Methods Mol. Biol. 262, (2004).

57. Dean, S. et al. A toolkit enabling efficient, scalable and reproducible gene tagging in trypanosomatids. Open Biol. 5, (2015).

58. Laemmli, U. K. Cleavage of structural proteins during the assembly of the head of bacteriophage T4. Nature (1970) doi:10.1038/227680a0.

59. Wittig, I. \& Braun, H. P. Blue native PAGE : Abstract : Nature Protocols. Nat. Protoc. (2006).

60. Bligh, E. G. \& Dyer, W. J. A rapid method for total lipid extraction. Biochem. Cell Biol. (1959) doi:10.1139/059-099. 


\section{PQQ Repeat}

TbEMC1 (Tb927.4.590) 89.5 kDa

TPR like

TbEMC2 (Tb927.7.6260) 35.7 kDa

DUF

TbEMC3 (Tb927.10.4760) $26.8 \mathrm{kDa}$

DUF TbEMC4 (Tb927.6.2600) 15.8 kDa

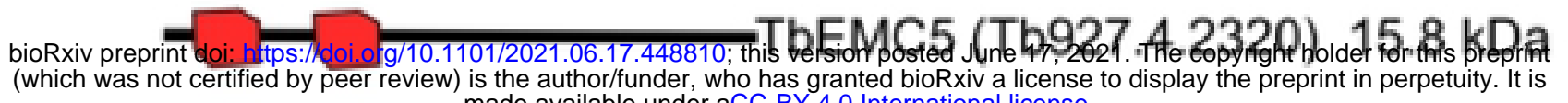

made available under aCC-BY 4.0 International license.

RAB-TbEMC6 (Tb927.11.17010) $11.8 \mathrm{kDa}$

Carb bd DUF TbEMC7 (Tb927.7.1290) $26.8 \mathrm{kDa}$

TbEMC8 (Tb927.7.1570) $30.1 \mathrm{kDa}$

TbEMC10 (Tb927.1.860) 29.7 kDa

Figure 1. Predicted domain structure of individual subunits of the TbEMC complex drawn to scale. Predicted transmembrane domains are indicted in red and unique domains are indicated in colors. PQQ, pyrrolo-quinoline quinone repeat; TPR like, tetratricopeptide-like helical domain; DUFs, domains of unknown function; RAB, Rab5-interacting protein family; Carb bd, carbohydrate-binding like fold. 

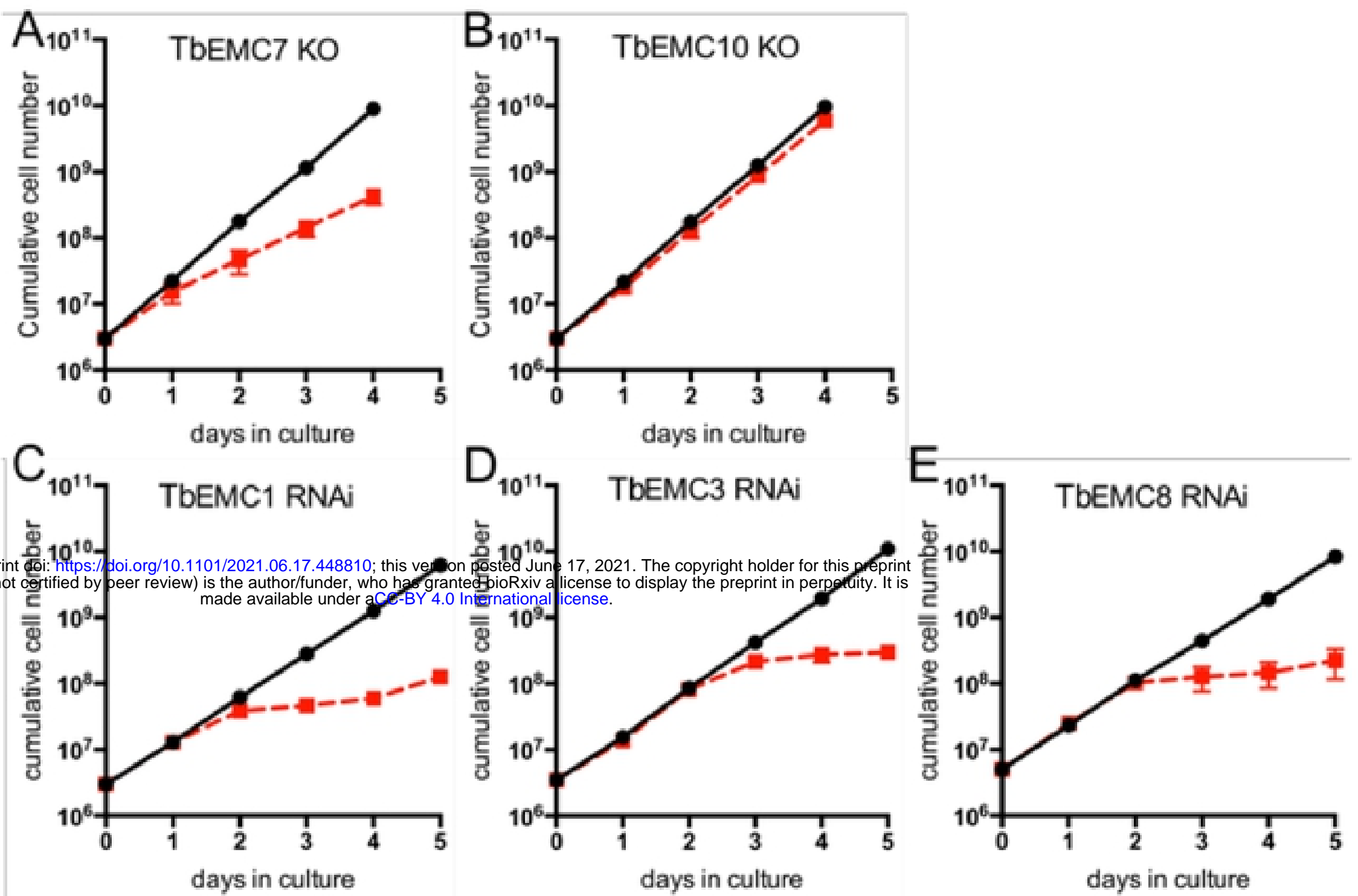

Figure 2. Growth of T. brucei procyclic forms. A, B. Growth of TbEMC7-KO (A) and TbEMC10KO (B) parasites (red symbols) was recorded in parallel to growth of parental parasites (black symbols). C-E. Parasites were cultured in the absence (black symbols) or the presence (red symbols) of tetracycline to maintain or ablate, respectively, expression of TbEMC1 (C), TbEMC3 (D) or TbEMC8 (E). All data points represent mean values \pm standard deviations from 3 independent experiments. For some data points, the size of the symbol is larger than the standard deviation. 


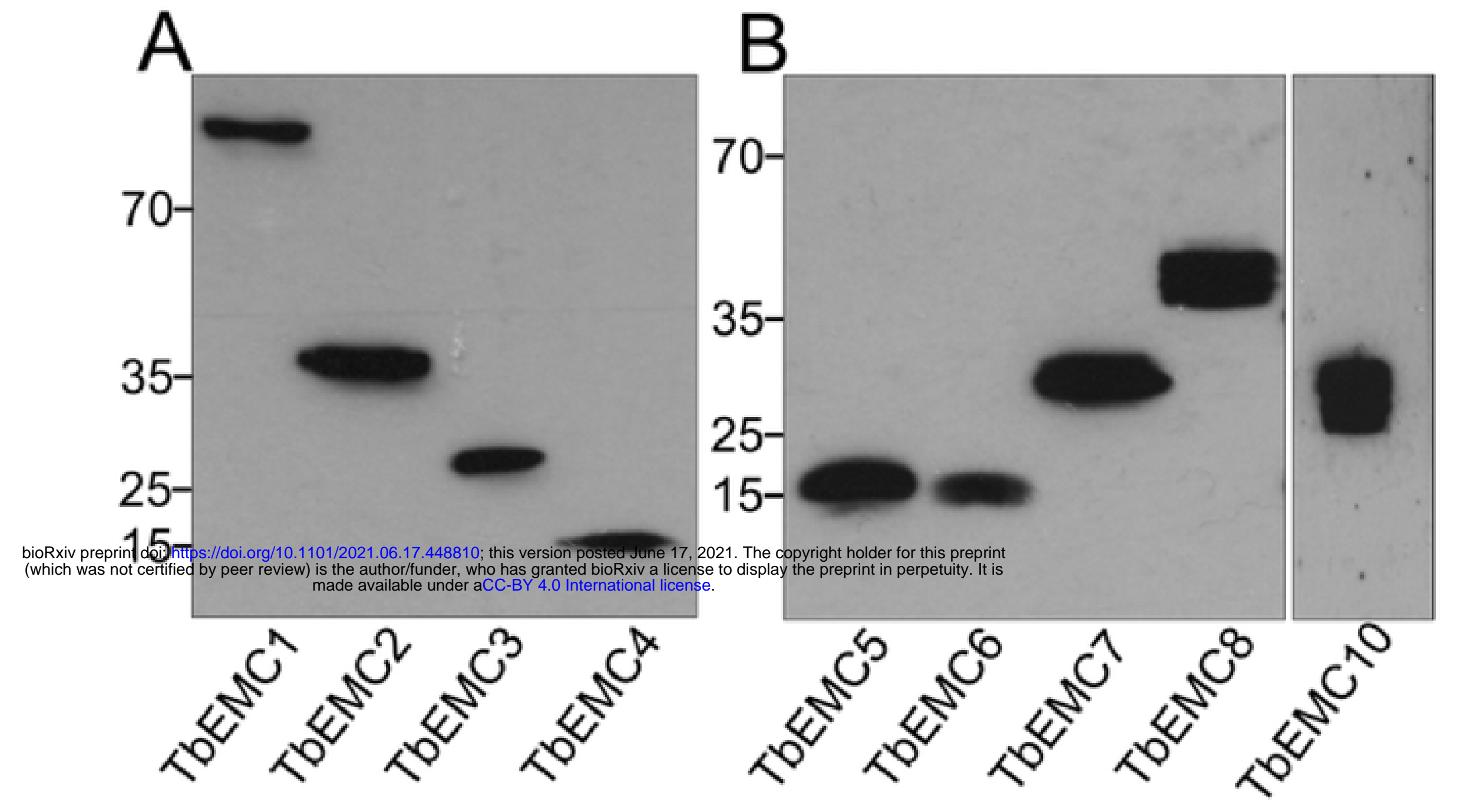

Figure 3. Expression of in situ-tagged TbEMCs. Protein extracts from parasites expressing HAtagged (A) or cMyc-tagged (B) TbEMC proteins were analyzed by SDS-PAGE and immunoblotting using anti-HA (A) or anti-cMyc (B) antibodies, in combination with the corresponding secondary antibodies. Molecular mass markers (in $\mathrm{kDa}$ ) are indicated in the left margins. 


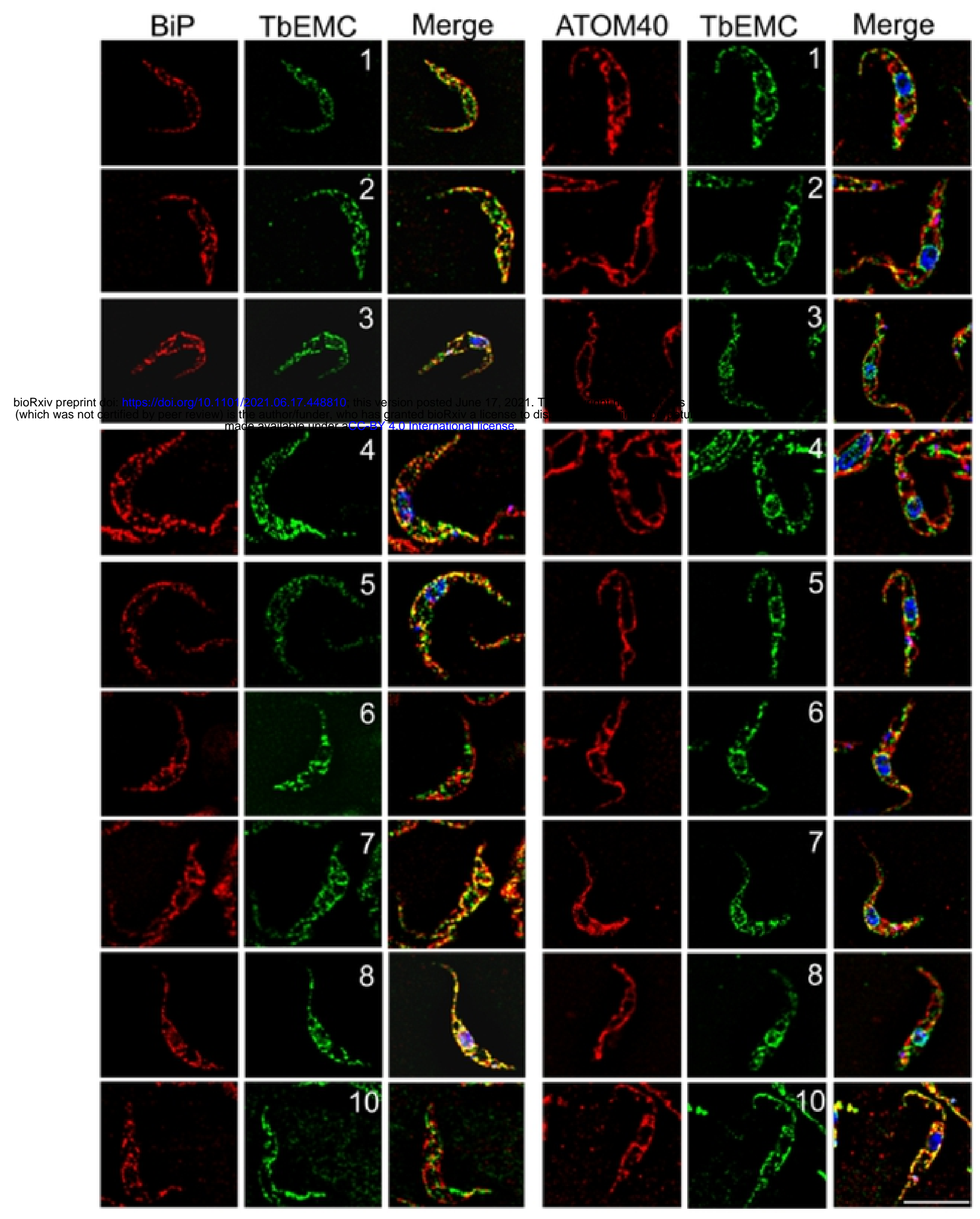

Figure 4. Localization of the TbEMCs. Parasites expressing in situ-tagged TbEMCs were fixed and analyzed by immunofluorescence microscopy using the corresponding first and second antibodies to visualize the TbEMC proteins (in green; middle panels; the numbers refer to the 
individual TbEMC proteins) and in combination with antibodies against BiP or ATOM40 (in red; left panels). DNA was stained with DAPI in the merge (in blue; right panels). Scale bar = $5 \mu \mathrm{m}$. 

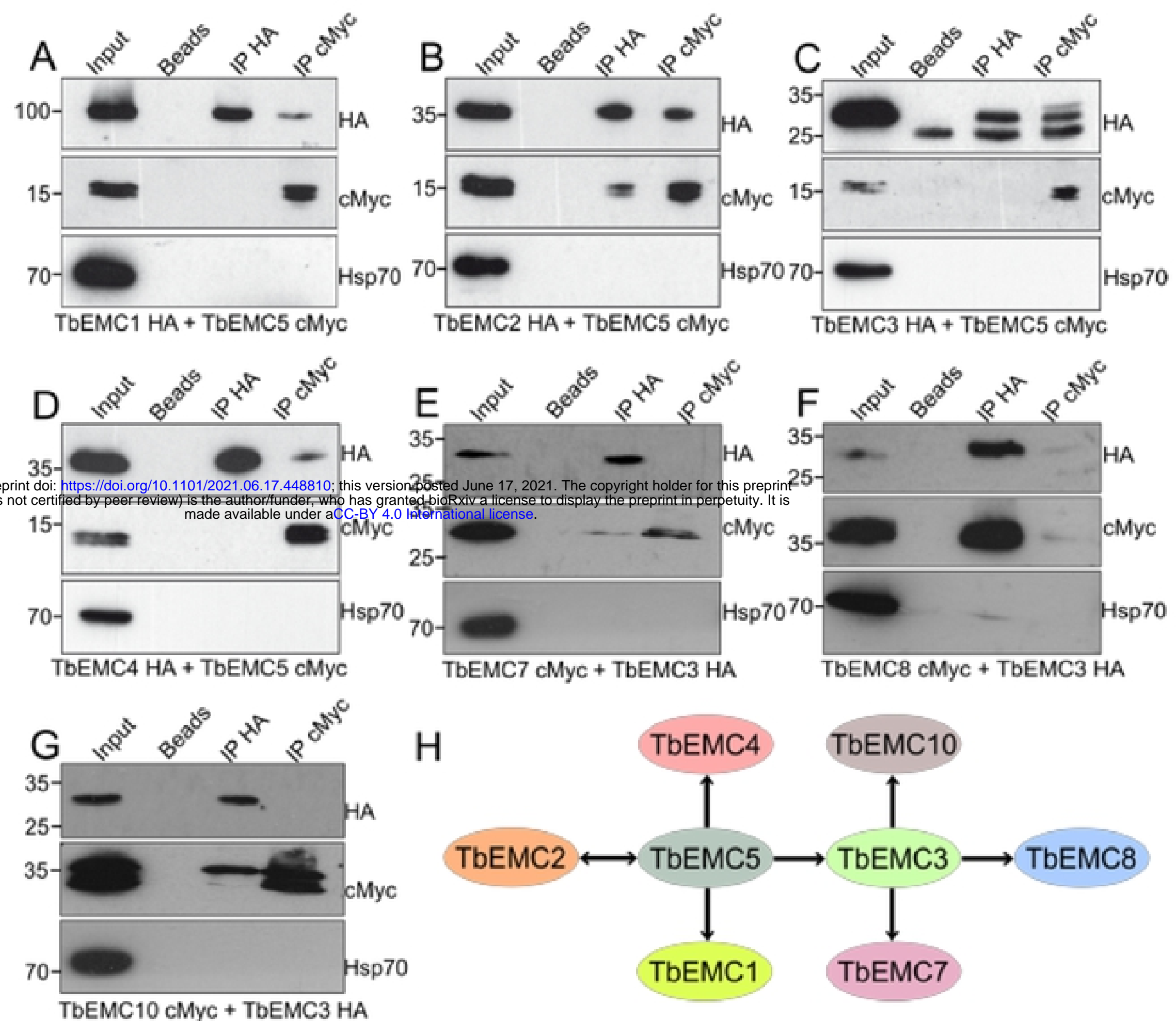

$$
\text { I }
$$
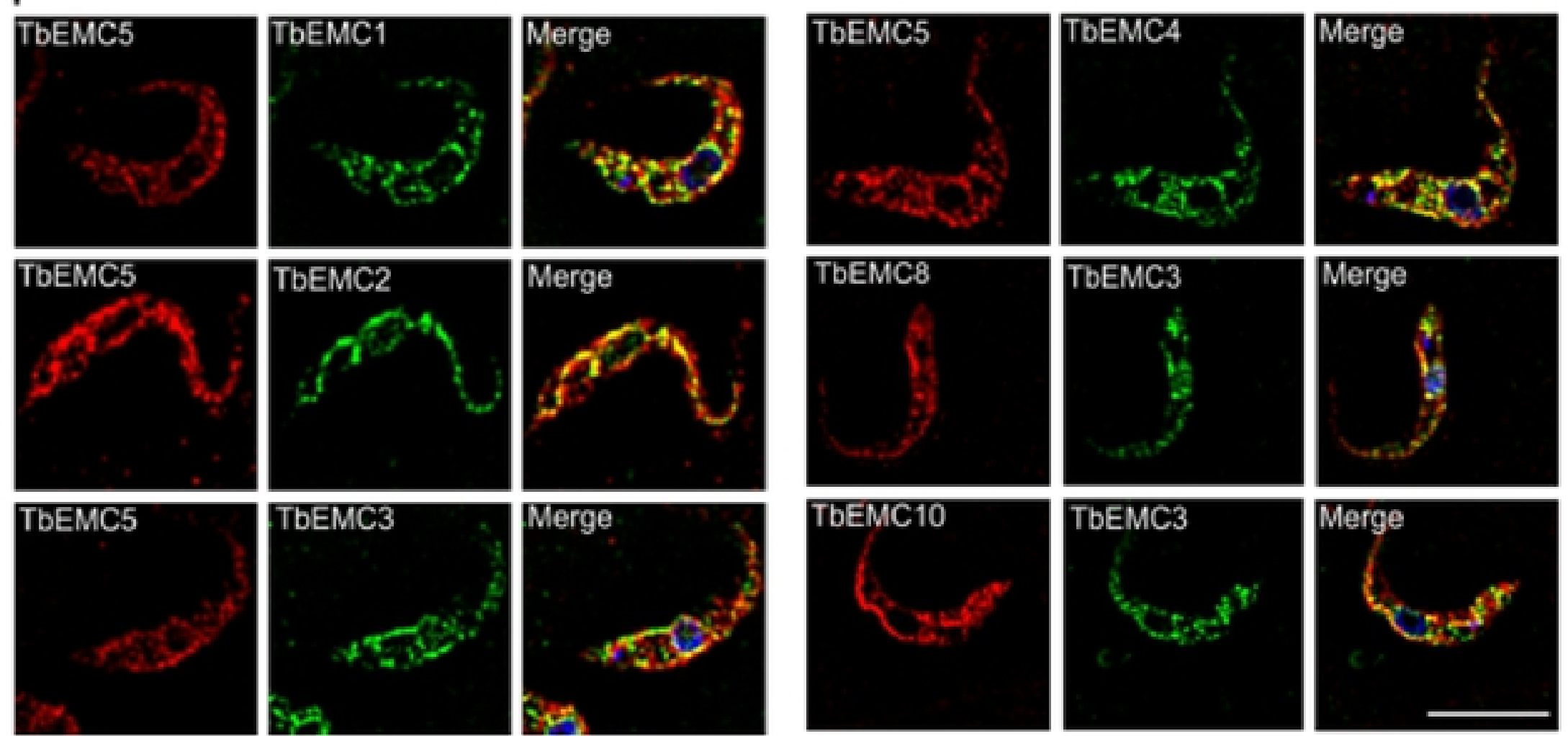
Figure 5. Interactions between individual TbEMCs. A-G. Proteins from parasites co-expressing differently tagged TbEMC proteins (indicated below each panels) were immunoprecipitated using TbEMC5-cMyc (A-D) or TbEMC3-HA (E-G) as bait. Proteins from the input, washed beads, and both immunoprecipitations (IP) were analyzed by SDS-PAGE and immunoblotting using anti-HA or anti-cMyc antibodies as indicated, in combination with the corresponding second antibodies. Heat shock protein $70(\mathrm{Hsp} 70)$ was used as negative control and visualized

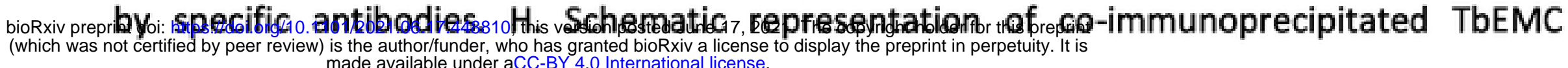

subunits using TbEMC5 and TbEMC3 as baits. Double headed arrows indicate reciprocal coimmunoprecipitation. I. Parasites co-expressing different pairs of in situ-tagged TbEMCs (indicated in the individual panels) were fixed and analyzed by immunofluorescence microscopy using the corresponding first and second antibodies to visualize the two TbEMC proteins (in red for cMyc; in green for HA; left two panels). DNA was stained with DAPI in the merge (in blue; right panels). Scale bar $=5 \mu \mathrm{m}$. 

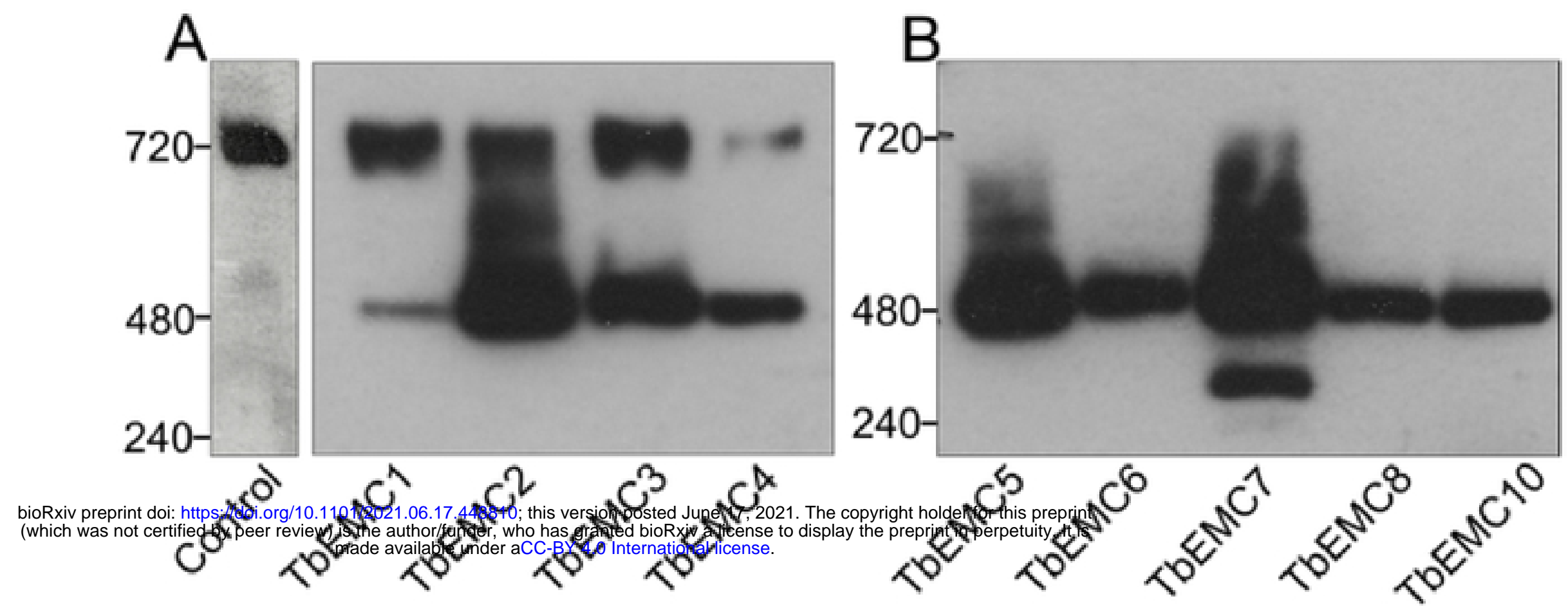

Figure 6. Analysis of TbEMC high molecular mass complexes. A, B. Crude membrane fractions from parasites expressing in situ-tagged TbEMC proteins (indicated below the panels), or from parental cells (control) were analyzed by native PAGE and visualized after immunoblotting using anti-HA (A) or anti-cMyc (B) antibodies. Molecular mass markers (in kDa) are indicated in the left margins. 


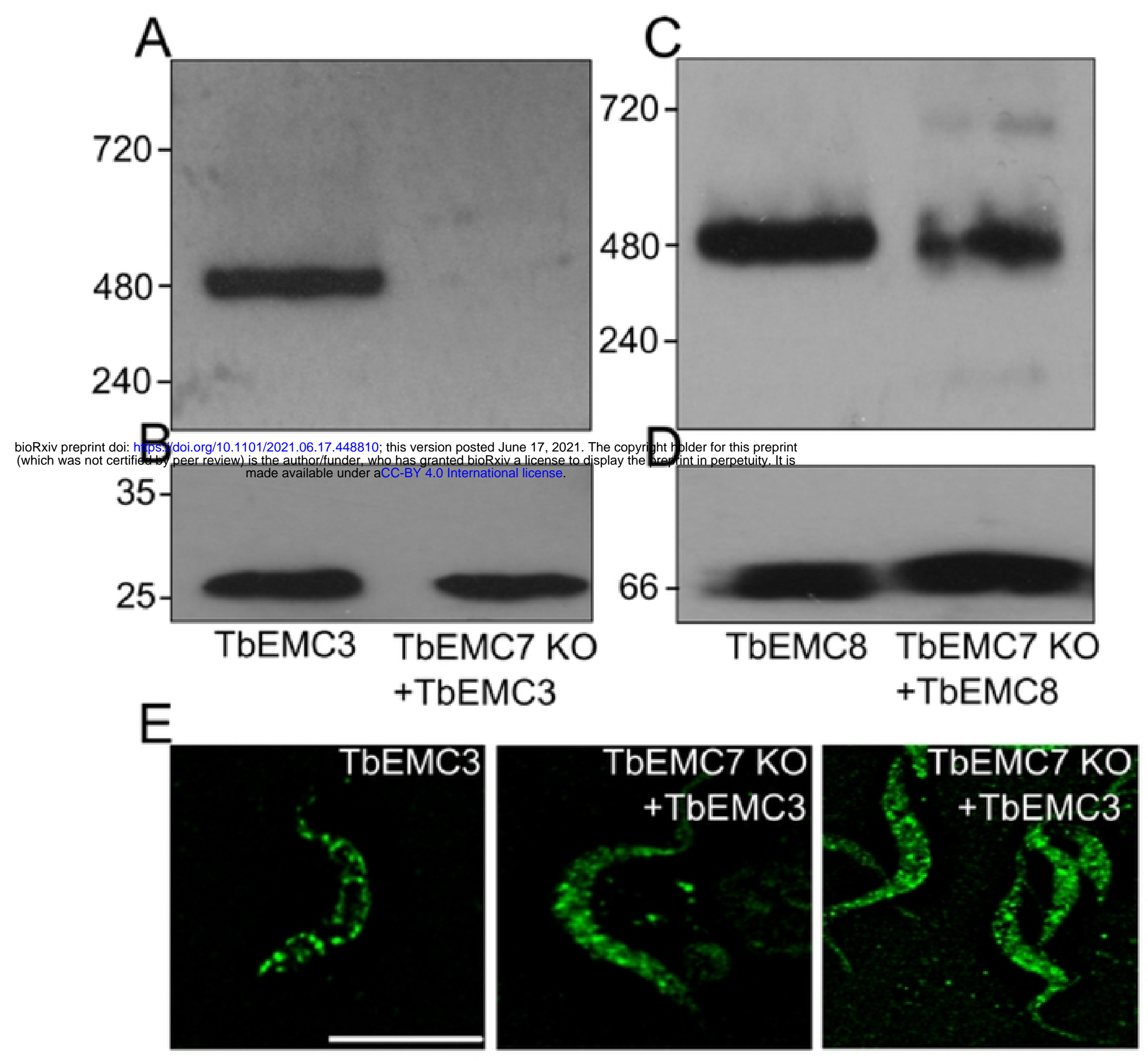

Figure 7. Analysis of TbEMC integrity. A-D. Crude membrane fractions from control cells ( $A$;

EMC3; C: EMC8) and TbEMC7-KO parasites (A: EMC7 KO + EMC3; C: EMC7 KO + EMC8) expressing in situ-tagged TbEMC3-cMyc or TbEMC8-cMyc, respectively, were analyzed by native PAGE $(\mathrm{A}, \mathrm{C})$ and immunoblotting using anti-cMyc antibody and visualized by the corresponding second antibody. The samples were also analyzed by SDS-PAGE and immunoblotting using anti-cMyc antibody (B) or an antibody against T. brucei ADP/ATP carrier 1 (AAC1; as loading control) (D) and visualized by the corresponding second antibodies. 
Molecular mass markers (in kDa) are indicated in the left margins. E. Control cells (EMC3) and TbEMC7-KO parasites (EMC7 KO + EMC3) expressing in situ-tagged TbEMC3-cMyc were fixed and analyzed by immunofluorescence microscopy using anti-cMyc antibody and the corresponding second antibody. Scale bar $=5 \mu \mathrm{m}$. 
A

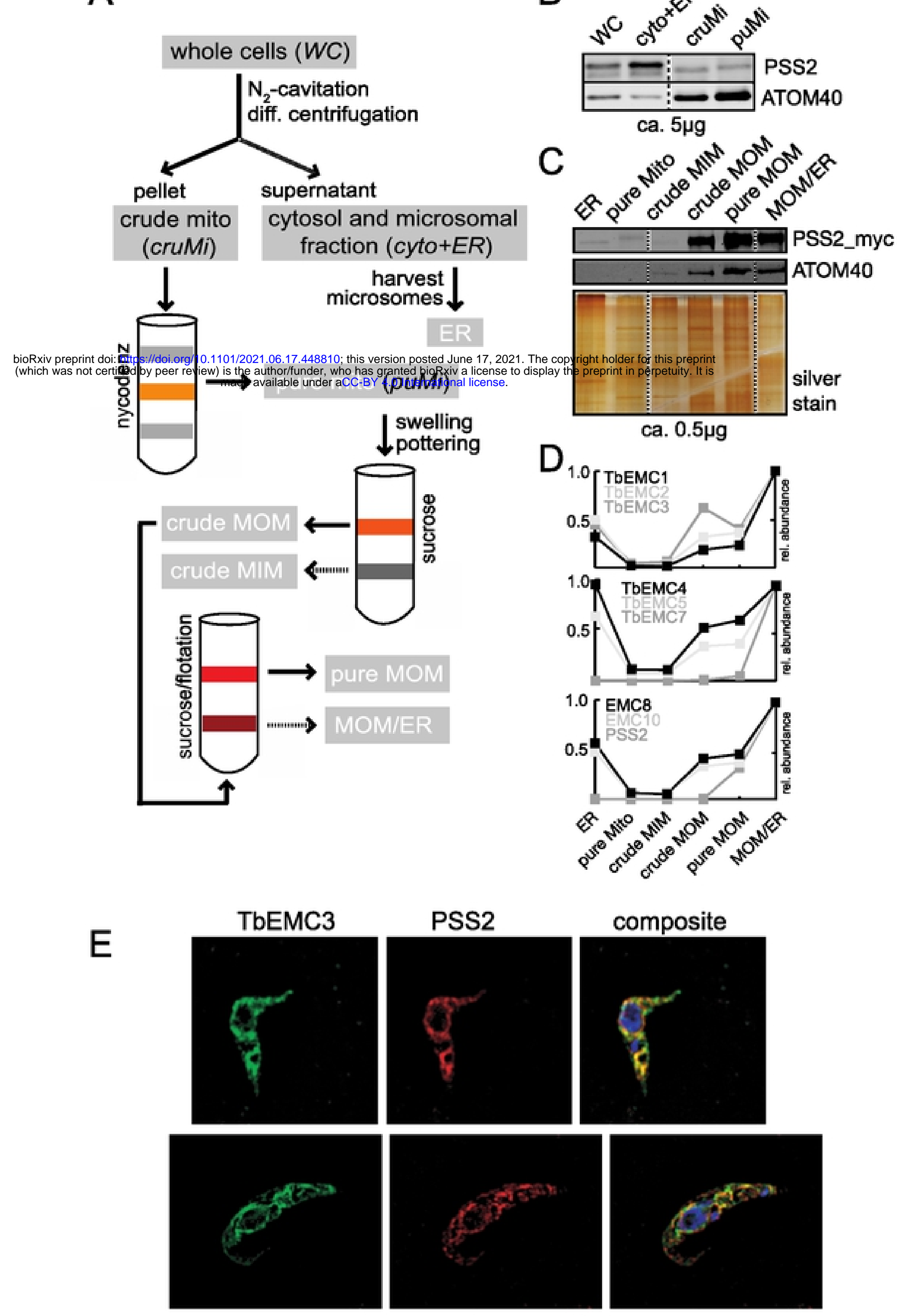


Figure 8. TbEMCs at the mitochondrial-ER interface. A. Overview of the purification method for mitochondrial vesicles ${ }^{47}$ and the mitochondrial outer membrane ${ }^{48}$. B-C. SDSPAGE/immunoblot analyses of the four fractions whole cell (WC), cytosol and microsomal ER fraction (cyto and ER), crude (cruMi) and pure mitochondria (puMi). Abbreviations indicated in black italics in the sketch are analyzed in panel (B), fractions in white text are analyzed in panel (C). D. Protein abundance profiles reproduced from Niemann et al., 201348. E. Parasites

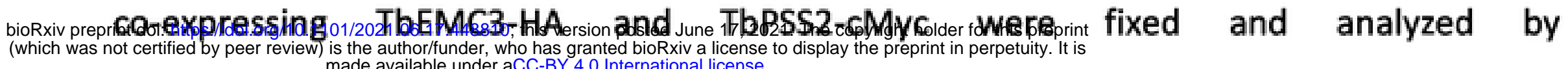

immunofluorescence microscopy using the corresponding first and second antibodies. DNA was stained with DAPI in the composite (in blue; right panels). Scale bar $=5 \mu \mathrm{m}$. 

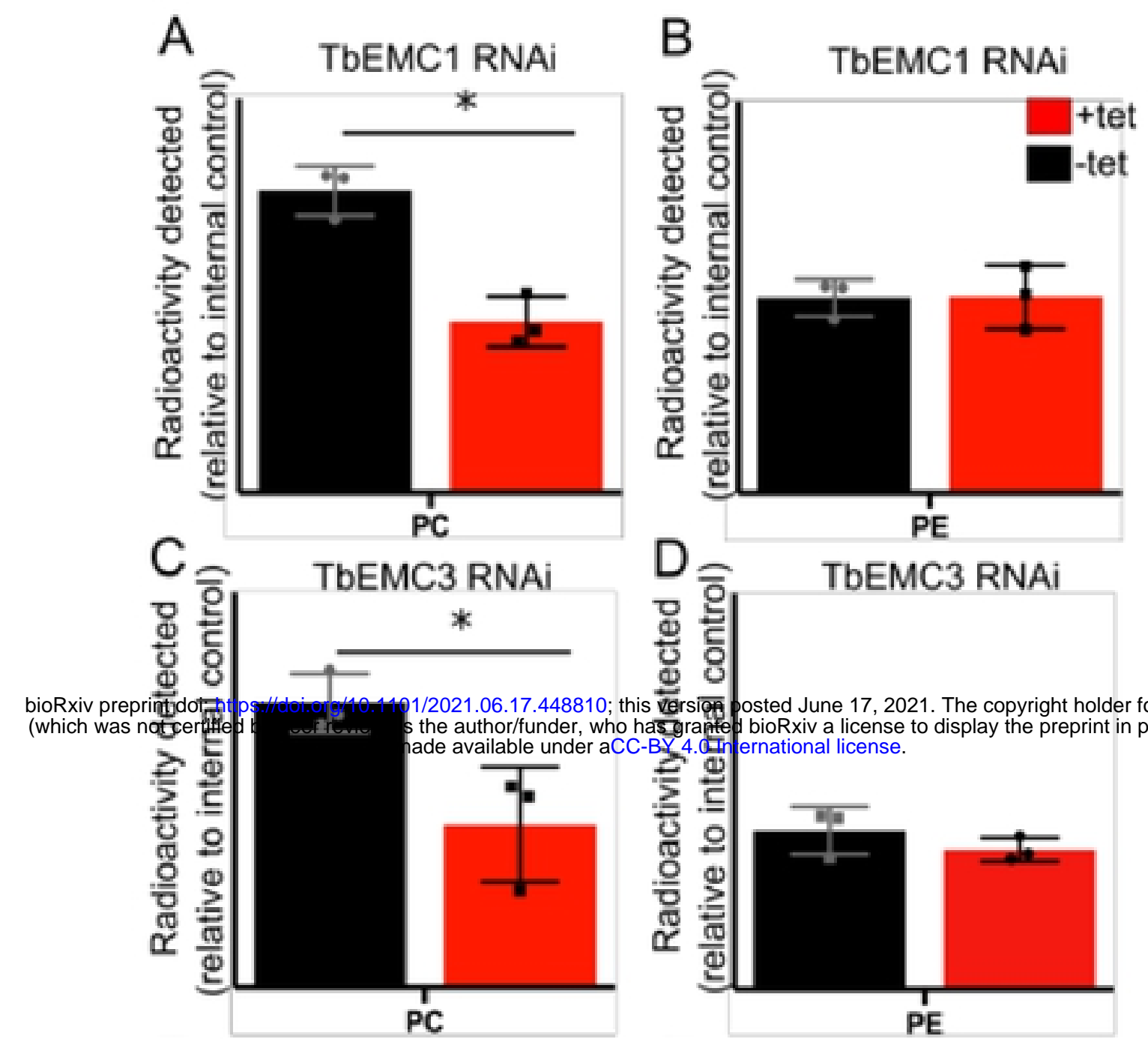

D oิ TbEMC3 RNAi
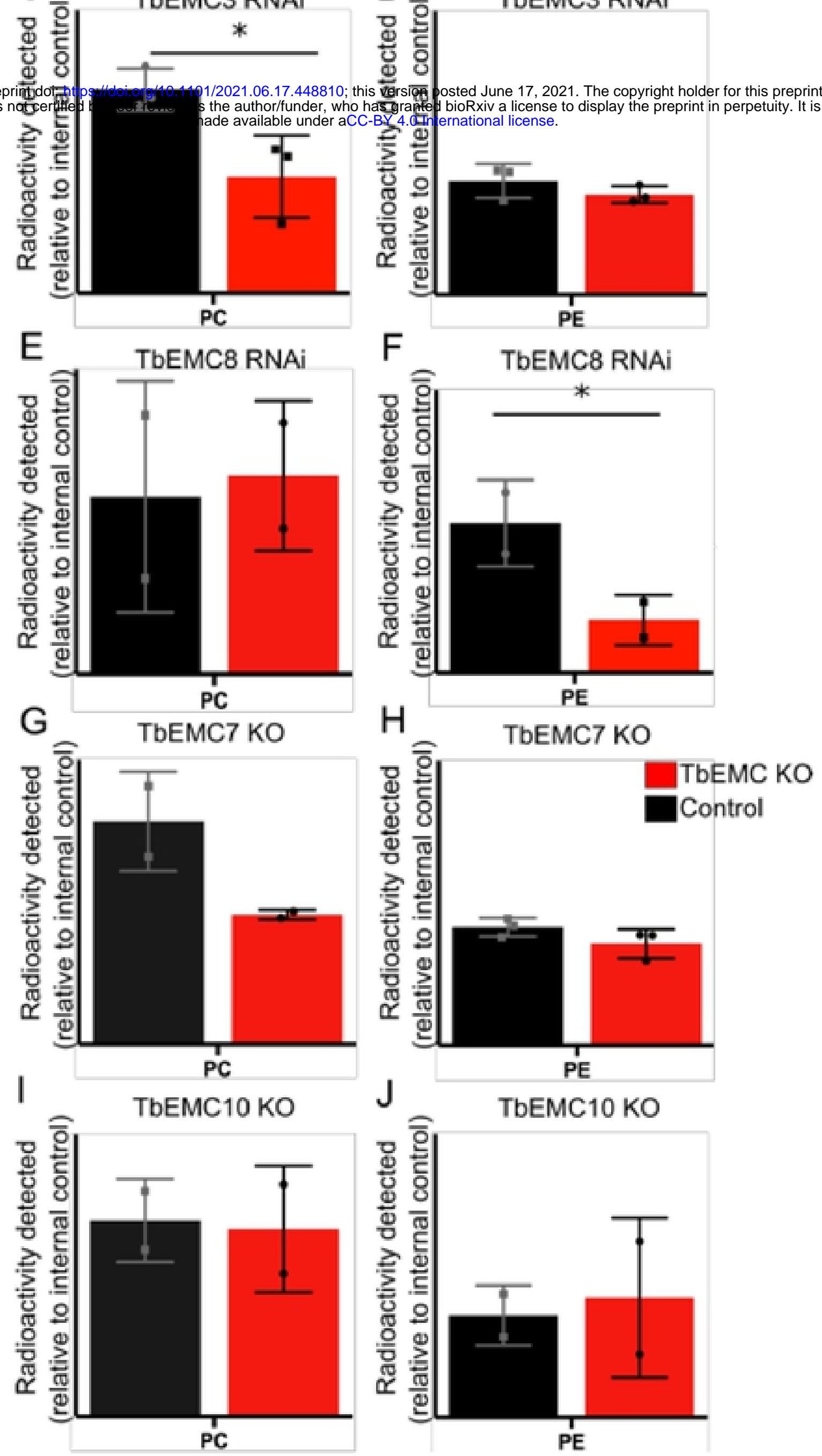
Figure 9. Analysis of $\left[{ }^{3} \mathrm{H}\right]-\mathrm{PC}$ and $\left[{ }^{3} \mathrm{H}\right]-\mathrm{PE}$ formation in TbEMC-depleted parasites. Parasites cultured in the absence (black) or the presence (red) of tetracycline to maintain or ablate, respectively, TbEMC expression (A-F), or parasites lacking individual TbEMC proteins (G-J), were labeled with $\left[{ }^{3} \mathrm{H}\right]$-choline or $\left[{ }^{3} \mathrm{H}\right]$-ethanolamine, in combination with $\left[{ }^{3} \mathrm{H}\right]$-inositol as internal standard, to measure de novo formation of phosphatidylcholine (PC) or

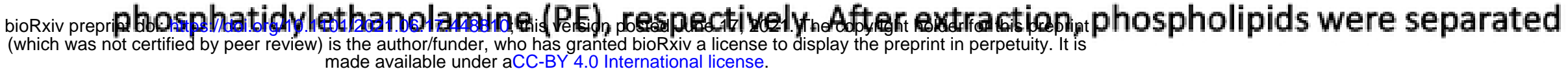

by TLC and the amounts of radioactivity in individual peaks were quantified by radioisotope scanning. The data points are from independent experiments and are expressed relative to control values (means \pm standard deviations). The asterisks indicate statistical significance $(\mathrm{P}<0.05)$. 